CLINICAL STUDY

\title{
Circulating nerve growth factor levels in relation to obesity and the metabolic syndrome in women
}

\author{
Mònica Bulló ${ }^{1}$, Muhammad R Peeraully ${ }^{2}$, Paul Trayhurn ${ }^{2}$, J Folch ${ }^{3}$ and Jordi Salas-Salvadó ${ }^{1,4}$ \\ ${ }^{1}$ Unitat de Nutrició Humana, Facultat de Medicina i Ciències de la Salut de Reus, Universitat Rovira i Virgili, 43201 Reus, Spain, ${ }^{2}$ Obesity Biology Unit, \\ School of Clinical Sciences, University of Liverpool, Liverpool, L69 3GA UK, ${ }^{3}$ Unitat de Bioquímica, Facultat de Medicina i Ciències de la Salut de Reus, \\ Universitat Rovira i Virgili, 43201 Reus, Spain and ${ }^{4}$ Unitat de Nutrició Humana, Servei de Medicina, Departament de Bioquímica i Biotecnologia, Facultat \\ de Medicina i Ciències de la Salut, Interna Hospital Universitari Sant Joan, C/Sant Llorenç 21, 43201 Reus, Spain
}

(Correspondence should be addressed to J Salas-Salvadó; Email: jordi.salas@urv.cat)

\begin{abstract}
Objective: Neurotrophins (NTs) could be involved in the development and progression of inflammatory and immune diseases. Because obesity and the metabolic syndrome (MetSyn) are related to a lowgrade systemic inflammation, plasma NT levels (neurotrophinemia) could play an important role in the ethiopathogenic mechanisms underlying these metabolic derangements. This is the first study evaluating the plasma NT levels in a group of women with obesity and MetSyn, and also the adipose tissue nerve growth factor (NGF) expression in a small group of them.

Methods: Included were 146 adult women with different degrees of adiposity, with or without MetSyn. Plasma NT levels were measured. NGF expression was analyzed in s.c. adipose tissue of a subgroup of morbidly obese and normal-weight females.

Results: NGF plasma levels were 1.4-fold higher in overweight and obese subjects. Plasma NGF was, however, lower in a group of morbidly obese subjects than in overweight or obesity, but it remained elevated relative to the normal-weight group. Plasma NGF was significantly correlated with body mass index (BMI), percentage body fat, and waist circumference in non-morbidly obese subjects. NGF was positively related to inflammatory markers. NT3 and brain-derived neurotrophin factor seem to be more related to lipid profile than to BMI, adipose tissue distribution, or peripheral inflammatory markers. Subjects with type 2 diabetes, abdominal fat distribution, or the MetSyn showed significantly higher levels of NGF. The MetSyn was the only independent predictor of the variability observed in the NGF plasma values.

Conclusion: NGF is upregulated in obesity, type 2 diabetes, and the MetSyn. Whether this NT may contribute to inflammation and the metabolic derangements associated with body weight gain remains to be elucidated.
\end{abstract}

European Journal of Endocrinology 157 303-310

\section{Introduction}

White adipose tissue (WAT) is now recognized as an important endocrine organ with a high capacity to synthesize and secrete several hormones, together with multiple other protein signals and factors (1-3). These adipokines are involved in a range of functions, including appetite and energy balance, lipid metabolism, insulin sensitivity, and vascular homeostasis, as well as immunity and inflammation. Growing evidence indicates an important role for low-grade chronic inflammation as a triggering factor in the origin and maintenance of the diseases associated with obesity, particularly insulin resistance and cardiovascular disease, both of which are features of the metabolic syndrome (MetSyn). A variety of adipokines linked to inflammation, including tumour necrosis factor (TNF) $\alpha$, interleukin (IL)-1 $\beta, \quad$ IL-6, IL-8, IL-10, haptoglobin, leptin, and adiponectin, are released from adipose tissue into the circulation (4). The circulating levels and adipose tissue expression of these various inflammation-linked adipokines are related to BMI and body fat, and in some cases to derangements of glucose and lipid metabolism. For example, the expression and circulating level of TNF $\alpha$, IL-6, haptoglobin, and leptin are increased with obesity and diabetes (5-7), while adiponectin falls $(8,9)$.

The neurotrophins (NTs) have also been considered as potential adipokines, with possible links to the MetSyn and other inflammatory-related diseases $(10,11)$. The best known NT, nerve growth factor (NGF), has recently been shown to be directly secreted from both murine and human adipocytes in cell culture, indicating that it is indeed an adipokine $(12,13)$. Since NGF was discovered half a century ago (14), several other factors with similar structure and functions have been identified - NT3, 
NT4/5, and brain-derived neurotrophin factor (BDNF) which form the family of NTs (15). Initially, the NTs were identified as important factors in the functioning of the nervous system, being essential for the development and survival of neuronal cells $(16,17)$. In recent years, several studies have provided evidence that NTs, particularly NGF, act not only on cells of the peripheral and $\mathrm{CN}$, but also on the immune and endocrine systems (18). The production and release of NTs by immune and endocrine cells is also now recognized (19).

NTs are present constitutively in the blood of rodents and humans, and the levels increase during stress and certain autoimmune diseases. Several authors have reported high plasma levels of NGF, BDNF, and NT3 in patients with rheumatoid arthritis, systemic lupus erythaematosis, allergic disease, or asthma $(20,21)$. Higher plasma levels of these NTs have also been described in type 1 diabetic patients (22). Other studies indicate elevated NGF production and release associated with an increase in several proinflammatory cytokines, such as TNF $\alpha$ and IL-1 $\beta(23,24)$. We have demonstrated a marked stimulation of NGF expression and secretion in murine and human adipocytes by TNF $\alpha$, prostaglandin $\mathrm{PGD}_{2}$ and $\mathrm{J}_{2}$ series prostaglandins $\mathrm{PGJ}_{2}$, and $\Delta^{12}-\mathrm{PGJ}_{2}(25)$, emphasizing the link between this NT and inflammatory processes. Such a link is further supported by the inhibitory effect of dexamethasone and a peroxisome proliferator-activated receptor $\gamma$ agonist on NGF expression and secretion by adipocytes, each having an anti-inflammatory action (12). Interestingly, studies on a small group of patients with cardiovascular disease or the MetSyn have reported a systemic NT deficit in the case of NGF and BDNF (26-28).

The aim of the present study is to examine whether the circulating levels of NGF are linked to body mass index (BMI), diabetes, dyslipidemia, and the MetSyn in a group of 146 adult women.

\section{Materials and methods}

\section{Subjects}

A group of 146 women were consecutively recruited from the outpatient clinic of the University Hospital of Sant Joan (Catalunya, Spain). Subjects were included if they were women aged between 18 and 65 years, with or without obesity or MetSyn, after giving written informed consent to participate.

The subjects were defined as overweight or obese when BMI was between 25 and $40 \mathrm{~kg} / \mathrm{m}^{2}$, and as morbidly obese when the BMI was $40 \mathrm{~kg} / \mathrm{m}^{2}$, according to the OMS criteria for obesity diagnosis and treatment (29). The control group consisted of non-diabetic healthy females with a BMI between 18.5 and $24.9 \mathrm{~kg} / \mathrm{m}^{2}$.

The presence of diabetes was confirmed when a previous diagnosis of type 2 diabetes mellitus had been performed or when fasting plasma glucose equal to or greater than $7 \mathrm{mmol} / \mathrm{l}$ was observed on two consecutive occasions. Dyslipidemia was defined by the criteria given in the third report of the National Cholesterol Education Program (ATP III; when patients showed two or more of these events: low-density lipoprotein (LDL) cholesterol $4.14 \mathrm{mmol} / \mathrm{l}$, high-density lipoprotein (HDL) cholesterol $1.03 \mathrm{mmol} / \mathrm{l}$, triglycerides $2.0 \mathrm{mmol} / \mathrm{l}$, total cholesterol $6.1 \mathrm{mmol} / \mathrm{l}$, or previous diagnosis of dyslipidemia). The MetSyn has been defined according to the ATP III criteria (30). All subjects were free of overt inflammatory or infectious diseases and none were receiving anti-obesity or anti-inflammatory drugs at the time of the study. Exclusion criteria were leukocyte counts higher than $11 \times 10^{9}$ cells $/ \mathrm{l}$, the presence of infectious, inflammatory, neoplastic or systemic diseases, hypothyroidism, or endocrine diseases other than diabetes. The study protocol was approved by the Hospital Ethics Committee.

\section{Measurements}

Height and weight were measured without shoes and with light clothes, and BMI was calculated as weight $(\mathrm{kg}) /$ height $(\mathrm{m})^{2}$. Waist circumference was measured midway between the lower rib margin and the iliac crest. Whole-body impedance at $50 \mathrm{kHz}$ was measured using a tetrapolar bioelectrical impedanciometer (HumanIm-Scan Dietosystem, Sabadell, Spain). Fat-free mass (FFM) was estimated using the gender-specific equations validated by Segal (31). Fat mass was estimated by the difference between body weight and FFM.

Venous blood samples were taken at $0800 \mathrm{~h}$ under overnight fasting conditions. Plasma was immediately separated by centrifugation at $900 \boldsymbol{g}$ for $10 \mathrm{~min}$ at $4{ }^{\circ} \mathrm{C}$ and aliquots were frozen at $-80{ }^{\circ} \mathrm{C}$ for subsequent analysis. Glucose, triglycerides, and lipid profile were assessed by the hospital's routine chemistry laboratory. Plasma levels of BDNF and NT3 were measured by specific ELISAs, with an intra-assay coefficient of variation less than $8.8 \%$ (Promega), and performed according to the manufacturer's instruction in 96-well microplates (Deltalab, Barcelona, Spain). Absorbance was measured at $450 \mathrm{~nm}$ with a Sunrise Reader microplate spectrophotometer (Tecan, Salzburg, Austria). NGF plasma levels were also measured using a commercial ELISA (R\&D Systems Inc., Minneapolis, MN, USA) with intra- and inter-assay coefficients of variation $<5 \%$. NGF concentrations were determined from the regression line for the NGF standard (ranging from 12.5 to $400 \mathrm{pg} / \mathrm{ml}$ ). The measured levels for NGF were corrected for mean recoveries of added mouse NGF $(140 \mathrm{pg} / \mathrm{ml})$, which were determined in each assay, this being a modified method previously described by other authors (32). Determinations of recovery, specific, and unspecific NGF binding were run for each plasma sample. Plasma insulin concentrations were measured by RIA (Amersham Pharmacia Biotech). Insulin 
resistance was measured from fasting plasma glucose and insulin concentrations using the HOMA1 method.

Serum C-reactive protein levels were assessed by a commercial ELISA kit (Alpha Diagnostic International, San Antonio, TX, USA), with a detection limit of $0.35 \mathrm{ng} / \mathrm{ml}$. The intra- and inter-assay coefficients of variation were less than 4.5 and $5 \%$ respectively. Immunoenzymometric assays were used to determine the levels of soluble TNF receptor (sTNFR) plasma levels (BioSource, Nivelles, Belgium). Leptin levels were assessed by RIA (Linco Research, St Louis, MO, USA). IL-6 was assessed by a high-sensitivity commercial ELISA (Amersham Pharmacia Biotech), with a detection limit of $0.1 \mathrm{pg} / \mathrm{ml}$. The intra- and inter-assay coefficients of variation were less than $10 \%$.

S.c. abdominal adipose tissue samples were obtained from six morbidly obese (BMI 40.1-49.3 kg/m ${ }^{2}$ ) and five normal-weight subjects $\left(23.5-26.5 \mathrm{~kg} / \mathrm{m}^{2}\right)$ by biopsy or elective surgical procedures (cholecystectomy, abdominal herniorrhaphy). Both groups were equivalent in age $(49.0 \pm 4.22$ vs $50.20 \pm 4.44$ respectively; $P=0.765)$. The anesthetic procedures were standardized and epinephrine was avoided. Total RNA was extracted from tissue samples with Tri-Reagent and treated with a DNA-free Kit (Ambion, Warrington, UK), in accordance with the manufacturer's instructions, to remove any contamination by genomic DNA. Human NGF mRNA levels were analyzed by relative quantitation with the $2^{-\Delta \Delta C t}$ method using real-time PCR with an ABI Prism 7700 instrument (Applied Biosystems, Foster City, CA, USA). All samples were normalized to values of $\beta$-actin, and the results were expressed as the fold change in $C_{\mathrm{t}}$ value of the obese group relative to the lean. Primer and Taqman probe sequences were as previously described (12); the primers and probes were synthesized commercially (Sigma-Genosys and Eurogentec, Southampton, UK respectively).

One microgram of total RNA was reverse-transcribed to cDNA in a $20 \mu$ reaction volume with anchored oligo(dT) primer using Reverse-iT First Strand Synthesis Kit (ABgene, Epsom, UK). Real-time PCR was performed in 96-well plates using a qPCR Core Kit (Eurogentec), according to the supplier's instructions, as previously described (25). Data were collected and analyzed with Sequence Detector software (Applied Biosystems).

\section{Statistical analysis}

Because of the lack of previous studies assessing the relationship between NTs and obesity or its comorbidities, we have carried out a study in women with obesity and MetSyn, designing a pilot study with a minimum sample from a statistical point of view. In future studies, sample size should be calculated to ensure adequate statistical power. Because levels of NTs, IL-6, PCR, and insulin concentrations were skewed, the values were log-transformed so as to approach a normal distribution and the geometric means are presented here. One-way
ANOVA with multiple comparisons and the Bonferroni post hoc test was used to explore differences between groups. Relationships between quantitative variables were assessed by Pearson's correlation coefficient. Covariance analysis was used to adjust plasma NT levels, and the significance of the differences between groups was assessed by the F-statistics. A stepwise multiple regression analysis was performed to identify the independent predictors of NTs. Statistical significance was set at $P<0.05$ and all analyses were performed using the SPSS/PC package.

\section{Results}

The anthropometric and biochemical parameters of the different groups of subjects are documented in Table 1. Because plasma NT levels were similar between overweight and obese females, and only 13 patients were overweight, we decided to present the results together. Plasma NGF levels were significantly increased in the group of overweight and obese patients, the levels being 1.4-fold $(P<0.016)$ than those of lean subjects (Table 1). The NGF plasma levels in the morbidly obese group patients tend to be higher than those in the normal-weight group, but the level was slightly lower than the overweight and moderately obese subjects $(P=0.480)$. Correlation analysis showed no significant relationship between NGF and the anthropometrical variables analyzed such as BMI $(r=0.024, P=0.775$, Fig. 1B), percentage of body fat $(r=0.074, P=0.396)$, abdominal fat distribution $(r=0.004, P=0.967)$, or age $(r=0.029, P=0.731)$. However, when morbidly obese subjects were excluded from the analysis, NGFs were correlated to BMI (Fig. 1A), percentage body fat $(r=0.251, P=0.022)$, and waist circumference

Table 1 General characteristics of the study groups.

\begin{tabular}{lccc}
\hline & $\begin{array}{c}\text { Normal weight } \\
(n=25)\end{array}$ & $\begin{array}{c}\text { Overweight- } \\
\text { obese }(n=66)\end{array}$ & $\begin{array}{c}\text { Morbidly } \\
\text { obese } \\
(n=55)\end{array}$ \\
\hline Age (years) & $36.4(7.9)$ & $48.4(10.3)^{\dagger}$ & $43.0(10.4)^{\star \ddagger}$ \\
BMl (kg/m $\left.{ }^{2}\right)$ & $22.3(1.7)$ & $33.7(3.9)^{\dagger}$ & $47.1(6.5)^{\dagger \ddagger \#}$ \\
Body fat $(\%)$ & $27.1(2.6)$ & $43.3(5.0)^{\dagger}$ & $50.9(1.5)^{\dagger \S}$ \\
Waist $(\mathrm{cm})$ & $77.7(6.8)$ & $103.6(11.9)^{\star}$ & $122.8(13.8)^{\dagger \S}$ \\
Glucose $(\mathrm{mM})$ & $4.8(0.3)$ & $6.4(1.8)^{\dagger}$ & $6.3(2.0)^{\dagger}$ \\
Total cholesterol $(\mathrm{mM})$ & $4.8(0.6)$ & $5.8(1.0)^{*}$ & $5.2(1.0)^{*}$ \\
HDL cholesterol $(\mathrm{mM})$ & $1.9(0.3)$ & $1.5(0.4)^{\dagger}$ & $1.4(0.3)^{\dagger}$ \\
LDL cholesterol $(\mathrm{mM})$ & $2.7(0.6)$ & $3.6(1.0)^{\dagger}$ & $3.1(0.8)^{\star \ddagger}$ \\
Triglycerides $(\mathrm{mM})$ & $0.7(0.3)$ & $1.5(0.8)^{\dagger}$ & $1.4(0.1)^{\dagger}$ \\
Plasma insulin $(\mu \mathrm{U} / \mathrm{ml})$ & $7.0(1.3)$ & $14.4(2.2)^{\star}$ & $28.2(2.1)^{\dagger \S}$ \\
HOMA IR & $1.0(1.7)$ & $3.8(2.2)^{\dagger}$ & $6.1(2.4)^{\dagger \ddagger}$ \\
NGF $(\mathrm{pg} / \mathrm{ml})$ & $56.2(1.5)$ & $77.6(1.9)^{*}$ & $69.2(2.1)$ \\
BDNF $(\mathrm{ng} / \mathrm{ml})$ & $4.0(2.6)$ & $4.6(2.9)$ & $2.4(3.4)^{\S}$ \\
NT3 (ng/ml) & $1.3(2.3)$ & $1.1(2.9)$ & $1.7(2.8)^{\ddagger}$ \\
& &
\end{tabular}

Values are given as mean (s.D.). ${ }^{\star} P<0.05$ and ${ }^{\dagger} P<0.001$ versus control normal-weight group; ${ }^{\ddagger} P<0.05$ and ${ }^{\S} P<0.001$ versus overweight-obese group. Insulin, HOMA, and neurotrophin values are expressed as the geometric mean. BMI, body mass index; NGF, nerve growth factor; BDNF, brain-derived neurotrophin factor; NT3, neurotrophin 3 . 
A
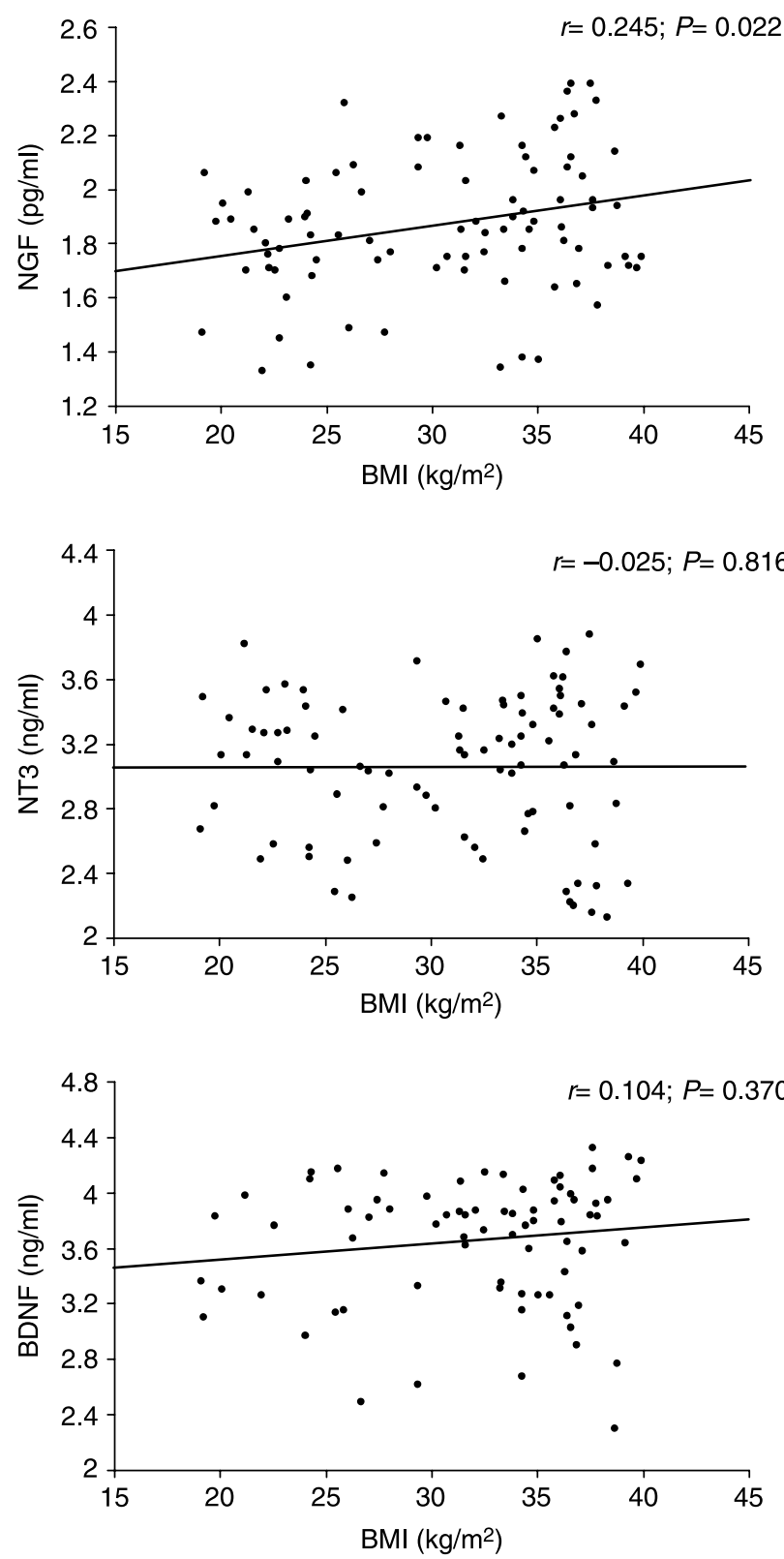

B
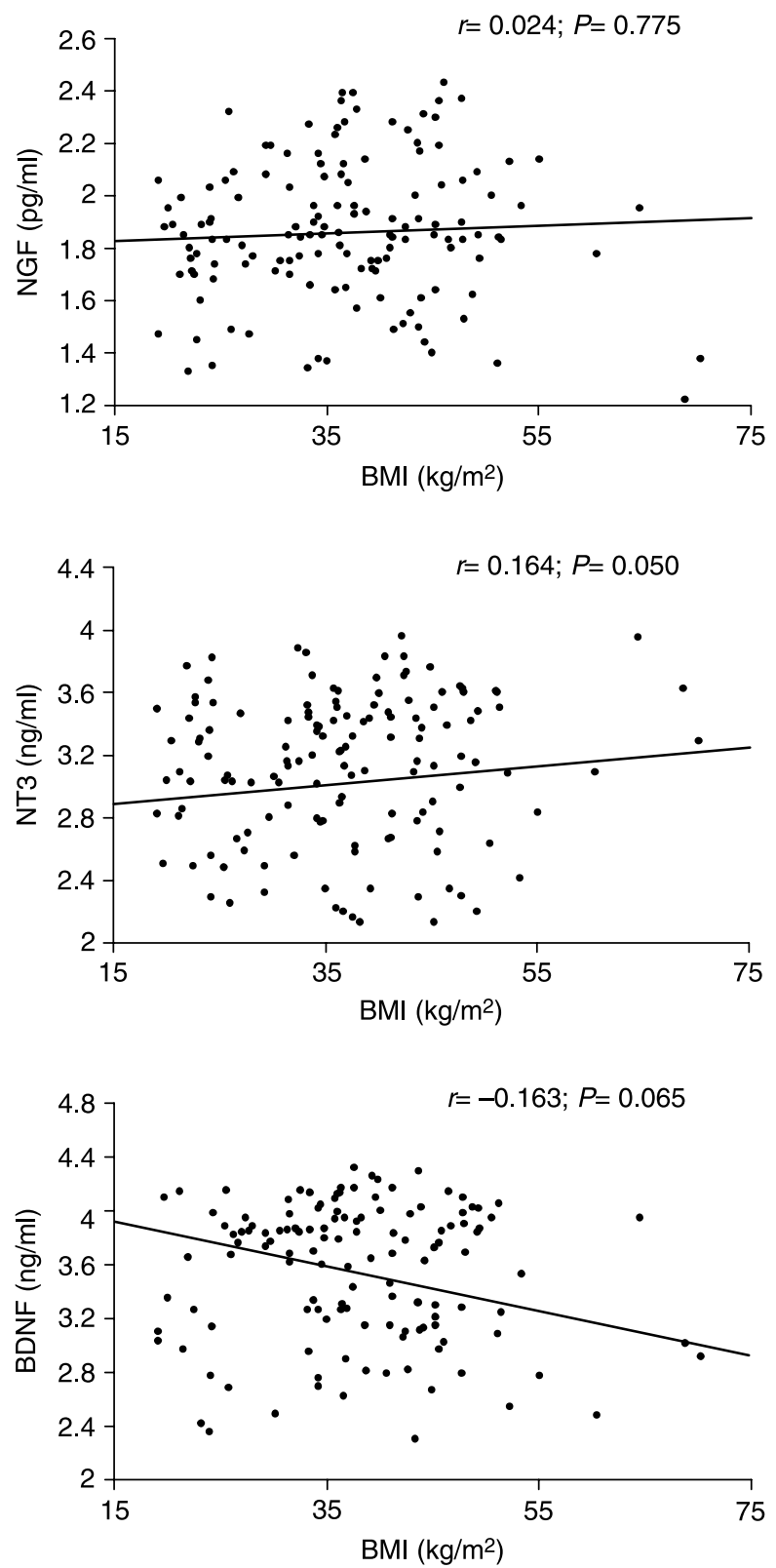

Figure 1 Plasma nerve growth factor (NGF), neurotrophin 3 (NT3), and brain-derived neurotrophin factor (BDNF) levels in relation to the degree of obesity in (A) the whole population or (B) patients with BMI up to $40 \mathrm{~kg} / \mathrm{m}^{2}$.

$(r=0.270 ; P=0.012)$, but not to age $(r=0.089$, $P=0.406)$. Further analysis indicated that plasma NGF was negatively related to HDL cholesterol and apolipoprotein A in the whole population (Table 2). The same pattern occurred even when extremely obese subjects were excluded from the analysis $(r=-0.258$, $r=-0.358 ; P<0.05$ respectively). Plasma NGF levels were also related to leptin levels, soluble TNFR1, and IL6 (Table 2), but not to the other inflammatory parameters analyzed (although the relationship with
CRP was at the limit of statistically significance $(P=0.06))$ when extremely obese were excluded.

Plasma NT3 and BDNF levels were also measured in the different groups. NT3 levels, but not BDNF, were positively related to BMI and waist circumference $(r=0.210, P=0.013)$ in the whole population but not when morbidly obese subjects were excluded (Fig. 1A) and tend to be higher in morbidly obese patients (Table 1). NT3 and BDNF seem to be most closely related to lipid profile than to adiposity degree, body fat 
Table 2 Relationship between neurotrophins and antropometrical or biochemical parameters in the whole population.

\begin{tabular}{lrcc}
\hline & Log NGF & Log NT3 & Log BDNF \\
\hline Glucose $(\mathrm{mM})$ & 0.094 & -0.066 & 0.043 \\
Total Cholesterol (mM) & 0.043 & $-0.277^{\dagger}$ & $0.208^{*}$ \\
HDL cholesterol (mM) & -0.124 & -0.045 & $0.194^{*}$ \\
VLDL cholesterol (mM) & 0.064 & -0.045 & 0.078 \\
LDL cholesterol (mM) & 0.099 & $-0.261^{\dagger}$ & 0.142 \\
TAG (mM) & 0.074 & -0.129 & $0.185^{\star}$ \\
Log HOMA IR & $0.232^{\dagger}$ & -0.027 & -0.022 \\
Leptin $(\mathrm{ng} / \mathrm{ml})$ & $0.195^{*}$ & 0.109 & -0.163 \\
STNFR1 (ng/ml) & $0.189^{\star}$ & -0.036 & $-0.172^{\star}$ \\
STNFR2 (ng/ml) & 0.065 & -0.046 & -0.156 \\
Log IL-6 (pg/ml) & $0.167^{\star}$ & 0.038 & $-0.320^{\dagger}$ \\
Log CRP (mg/dl) & 0.149 & 0.049 & -0.002 \\
\hline
\end{tabular}

${ }^{*} P<0.05$ and ${ }^{\dagger} P<0.001$. NGF, nerve growth factor; NT3, neurotrophin 3; BDNF, brain-derived neurotrophin factor.

distribution, or systemic inflammatory markers (Table 2).

When the study population was distributed in relation to the presence or absence of metabolic derangements and the criteria used in the definition of the MetSyn, a significantly higher plasma level of NGF was observed in patients with hyperglycemia and/or medication use and those affected by MetSyn, almost reaching statistical significance for the abdominal obesity distribution $(P=0.057)$ after adjusting for differences in age and BMI. NT3 and BDNF plasma levels were significantly lower in dyslipidemic patients. No significant differences in BDNF or NT3 were observed in relation to the presence or absence of MetSyn (Table 3). In a multiple linear regression analysis in which age, BMI, waist circumference, total cholesterol, HDL cholesterol, LDL cholesterol, tryglycerides, and HOMA IR were included as independent variables, HDL cholesterol was the only independent predictor of NGF, explaining $4.5 \%$ of the variance $(P=0.016)$. Using the same model, waist circumference and total cholesterol explained $14.3 \%$ of NT3 variability $(P<0.001)$ and tryglycerides and HDL cholesterol explained $10.6 \%$ of the variability observed in BDNF plasma levels $(P=0.002)$. When we consider the metabolic derangements associated with obesity and performed a new model including age, BMI, presence of dyslipemia, type 2 diabetes, and MetSyn as independent variables, the MetSyn explained 8.3\% of the variability observed in NGF plasma levels $(P=0.001)$, dyslipidemia and BMI explained $11.6 \%$ of NT3 variability $(P<0.001)$, and dyslipidemia alone explained $5.5 \%$ of the variability observed in BDNF plasma levels $(P=0.010)$.

The level of NGF mRNA was examined in s.c. adipose tissue of a small group of five normal-weight and six morbidly obese subjects. NGF mRNA was readily detected by RT-PCR in human adipose tissue, as in a previous study (11). Quantitative measurement by
Table 3 Plasma neurotrophin levels in relation to metabolic complications associated with obesity in the studied population.

\begin{tabular}{|c|c|c|c|}
\hline & $\begin{array}{c}\log \text { NGF } \\
(\mathrm{ng} / \mathrm{ml})\end{array}$ & $\begin{array}{c}\log \text { BDNF } \\
(\mathrm{ng} / \mathrm{ml})\end{array}$ & $\begin{array}{c}\log \text { NT3 } \\
(\mathrm{ng} / \mathrm{ml})\end{array}$ \\
\hline \multicolumn{4}{|l|}{ Abdominal obesity } \\
\hline Present $(n=113)$ & $72.44(2.0)$ & $3.23(0.03)$ & $1.2(0.03)$ \\
\hline Absent $(n=33)$ & $57.54(1.58)$ & $4.6(0.02)$ & $0.9(0.02)^{\star}$ \\
\hline \multicolumn{4}{|c|}{ Hyperglycemia and/or medication use } \\
\hline Present $(n=79)$ & $77.6(2.0)$ & $3.3(0.03)$ & $1.2(0.003)$ \\
\hline Absent $(n=67)$ & $63.1(1.8)^{*}$ & $3.7(0.03)$ & $1.4(0.002)$ \\
\hline \multicolumn{4}{|c|}{ Hypertriglyceridemia } \\
\hline Present $(n=38)$ & $77.62(2.2)$ & $4.3(0.003)$ & $0.8(0.002)$ \\
\hline Absent $(n=108)$ & $69.18(1.8)$ & $2.9(0.003)$ & $1.2(0.002)^{*}$ \\
\hline \multicolumn{4}{|c|}{ Low HDL cholesterol and/or medication use } \\
\hline Present $(n=39)$ & $74.1(2.5)$ & $2.5(0.003)$ & $1.3(0.002)$ \\
\hline Absent $(n=107)$ & $70.8(1.7)$ & $3.5(0.003)$ & $1.4(0.002)$ \\
\hline \multicolumn{4}{|c|}{ Hypertension and/or medication use } \\
\hline Present $(n=55)$ & $75.8(1.9)$ & $3.0(0.003)$ & $1.8(0.003)$ \\
\hline Absent $(n=91)$ & $66.1(1.9)$ & $4.0(0.003)$ & $1.2(0.002)^{\star}$ \\
\hline \multicolumn{4}{|l|}{ Metabolic syndrome } \\
\hline Present $(n=69)$ & $85.1(2.1)$ & $3.2(0.003)$ & $1.3(0.002)$ \\
\hline Absent $(n=77)$ & $60.2(1.7)^{\dagger}$ & $3.5(0.003)$ & $1.3(0.003)$ \\
\hline
\end{tabular}

Mean values are expressed as the geometric mean (s.D.). ${ }^{*} P<0.05$ and ${ }^{\dagger} P<0.001$ versus presence of metabolic diseases. NGF, nerve growth factor; BDNF, brain-derived neurotrophin factor; NT3, neurotrophin 3.

real-time PCR showed that there was a small but statistically significant $(P<0.05)$ increase in NGF mRNA level in the morbidly obese $(2.47 \pm 0.6$ arbitrary units) when compared with the normalweight subjects.

\section{Discussion}

This is the first study that evaluates the plasma levels of NTs in a group of women distributed over a wide range of adiposity, with or without MetSyn. We demonstrate that the circulating level of the first NT to be identified, NGF, is increased in overweight, obese, and morbidly obese subjects, and also in patients with MetSyn. We also show that plasma NT3 and BDNF levels are not significantly changed with obesity, although slightly higher levels of these NTs were observed in morbidly obese subjects. Moreover, a positive relationship was observed between peripheral levels of NTs and some of the measured circulating markers of inflammation commonly overproduced in obese patients or MetSyn.

Several studies over the last decade have implicated NTs in the pathogenesis of various inflammatory diseases $(20,21)$. The expression and production in immune cells of one or more NTs together with NT receptors have been described $(33,34)$. The effects of these NTs include cell survival or differentiation, and recently a regulatory effect on cytokine production or mediator release has also been identified (35).

Because inflammation is now considered to be an important feature of obesity, cardiovascular disease, and MetSyn, it has recently been suggested that NTs may be 
involved in these pathologies. Chaldakov and coworkers have hypothesized that a reduction in NT production could be implicated in the pathogenesis of obesity and related comorbidities, and reported that hyponeurotrophinemia was present in a group of patients with the MetSyn or cardiovascular disease and not in healthy subjects $(10,26-28)$. However, such a proposal is not in agreement with the relationship described between NTs and other inflammatory diseases $(20,21)$.

In contrast to these previous studies, our results show an increase in NGF plasma levels in patients with obesity or MetSyn. In parallel, for the first time, we report higher levels of NGF mRNA in s.c. adipose tissue from a small subgroup of morbidly obese females. It is important to note that NGF plasma levels increased 1.4 fold in morbidly obese patients in comparison with normal-weight healthy subjects, while adipose tissue NGF mRNA increased 2.4 times. NGF gene expression in human adipose tissue has previously been reported in a small group of morbidly obese volunteers, with a stronger signal in omental than in s.c. fat (12). Since waist circumference is considered as a good marker of omental adipose tissue depot size (36), the higher NGF expression levels described in omental fat may explain the positive relationship observed between NGF and waist circumference in our study.

Comparison of circulating NGF between obese and extremely obese patients, who have slightly lower NGF plasma levels than the morbidly obese, suggests that the levels essentially plateau with moderate obesity. Similar findings were made for the plasma levels of some other adipokines, such as leptin, in extremely obese patients (37-39). This suggests that the metabolic response, or metabolic adaptations, in the morbidly obese differ considerably from those of the general obese population. The relationship which we observed between NTs and the metabolic derangements associated with obesity or MetSyn differs from the results reported by Chaldakov et al., who reported lower levels of NGF in the vascular wall of human coronary arteries of atherosclerotic patients, as well as in the blood of patients with the MetSyn (10, 26-28). However, we find significant differences in plasma NGF levels in relation to hyperglycemia or medication use, abdominal fat distribution, and MetSyn. In our study, the presence of MetSyn appeared as independent predictor of NGF plasma variability.

It is difficult to compare our results with those reported by Chaldakov and co-workers. First, they did not describe the criteria they used to define MetSyn, and, secondly, their study population was smaller and there was a different proportion of men and women in the control subjects and the MetSyn group. Significant sex differences in plasma NGF have been described, the levels being higher in men than in women (40). Thus, the higher proportion of males in their control group than in the MetSyn patients may explain, at least in part, the differences observed by the authors. More recently, a neurotrophic hypothesis suggests that NTs have a different role in the early or generalized stage of MetSyn (11). Hristova and Aloe show that NGF levels are significantly higher in patients in the early stage of the MetSyn, while a hyponeurotrophinemia appears during the generalized stage. They suggest that hyperneurotrophinemia leads to a hyperactivation of the hypothalamic-pituitary-adrenal axis and exerts a compensatory mechanism against an inflammatory state. In the generalized stage of the MetSyn, these compensatory mechanisms fail and a hyponeurotrophinemia occurs leading to obesity, diabetes, atherosclerosis, and MetSyn. Our results are along the same lines as those reported for the early stage of MetSyn.

Inflammation mediates several effects that are important for cell survival, including changes in lipid metabolism leading to hyperlipidemia, which are partially mediated by cytokines such as TNF $\alpha$, IL-1, and IL-6 (41). NGF is reported to be able to raise serum free fatty acid and triglyceride levels after administration to rats (42). The relationship observed between lipid profile or inflammatory markers and NTs in our study suggests that NTs should be added to the list of cytokines and adipokines involved in the progression and maintenance of obesity and its comorbidities. However, little is known about the mechanisms by which NTs are involved in adipose tissue and lipid metabolism. Metabolic studies have been performed to elucidate the role of NTs in the regulation of whole body energy metabolism and in the control of eating behavior $(43,44)$. One study performed in isolated rat adipocytes has shown that NGF has an antilipolytic and lipogenic effect (45). Subsequently, an upregulation by NGF of LDL receptor-related protein has also been described in different cell types, including PC12 cells (46), which suggests a protective role of NGF in the atherosclerotic process.

The results of our study are consistent with the documented positive association between NGF- and inflammation-related diseases. Since NGF is synthesized and released by white adipocytes and the level of the mRNA was higher in a group of obese patients than in normal-weight subjects or in those with MetSyn, WAT may directly contribute to the increased circulating level of the NT in these metabolic derangements. In contrast to our observations with respect to NGF, we failed to find consistent differences in plasma levels of either NT3 or BDNF associated with obesity and MetSyn. This suggests that they may not be adipokines. However, these results need to be confirmed in an appropriately designed study since our study was limited by a small number of patients. Given the lack of data in the literature, we could not calculate the number of patients necessary for an adequately powered statistical analysis. Therefore, we performed this pilot study. Furthermore, we studied an outpatient population that may not be representative of the general community. 
In conclusion, the present study indicates that overweight and moderate obesity are related to an increase in the circulating level of NGF. However, because of the small increment of plasma NGF levels in overweight or obese subjects, whether this NT is involved in the changes in the inflammatory markers in the metabolic derangements requires further research.

\section{Acknowledgements}

This study was supported by the Fondo de Investigación Sanitaria (FIS 02/0550), Red de Centros RCMN (C03/08), Red de Grupos (G03/140), and Red de Grupos (RD06/0045) of the Instituto de Salud Carlos III, Madrid, Spain.

\section{References}

1 Trayhurn P \& Beattie JH. Physiological role of adipose tissue: white adipose tissue as an endocrine and secretory organ. Proceedings of the Nutrition Society 200160 329-339.

2 Rajala MW \& Scherer PE. Minireview: The adipocyte - at the crossroads of energy homeostasis, inflammation, and atherosclerosis. Endocrinology $2003 \mathbf{1 4 4} 3765-3773$.

3 Trayhurn P \& Wood IS. Adipokines: inflammation and the pleiotropic role of white adipose tissue. British Journal of Nutrition 200492 347-355.

4 Trayhurn P. Adipose tissue in obesity - an inflammatory issue. Endocrinology 2005146 1003-1005.

5 Bulló M, García-Lorda P \& Salas-Salvadó J. Plasma soluble tumor necrosis factor alpha receptors and leptin levels in normal-weight and obese women: effect of adiposity and diabetes. European Journal of Endocrinology 2002146 325-331.

6 Bulló M, García-Lorda P \& Salas-Salvadó J. Systemic inflammation, adipose tissue tumor necrosis factor, and leptin expression. Obesity Research 200311 525-531.

7 Chiellini C, Santini F, Marsili A, Berti P, Bertacca A, Pelosini C, Scartabelli G, Pardini E, Lopez-Soriano J, Centoni R, Ciccarone AM, Benzi L, Vitti P, Del Prato S, Pinchera A \& Maffei M. Serum haptoglobin: a novel marker of adiposity in humans. Journal of Clinical Endocrinology and Metabolism 200489 2678-2683.

8 Weyer C, Funahasshi T, Tanaka S, Hotta K, Matsuzawa Y, Pratley RE \& Tataranni PA. Hypoadiponectinemia in obesity and type 2 diabetes:close asociation with insulin resistance and hyperinsulinemia. Journal of Clinical Endocrinology and Metabolism 200186 1930-1935.

9 Bulló M, Salas Salvadó J \& García-Lorda P. Adiponectin expression and adipose tissue lipolytic activity in lean and obese women. Obesity Surgery 200515 382-386.

10 Chaldakov GN, Fiore M, Hristova MG \& Aloe L. Metabotrophic potential of neurotrophins: implication in obesity and related diseases? Medical Science Monitor 20039 HY19-HY21.

11 Hristova M \& Aloe L. Metabolic syndrome-neurotrophic hypothesis. Medical Hypotheses 200666 545-549.

12 Peeraully MR, Jenkins JR \& Trayhurn P. NGF gene expression and secretion in white adipose tissue: regulation in 3T3-L1 adipocytes by hormones and inflammatory cytokines. American Journal of Physiology. Endocrinology and Metabolism 2004287 E331-E339.

13 Wang B, Jenkins JR \& Trayhurn P. Expression and secretion of inflammation-related adipokines by human adipocytes differentiated in culture: integrated response to TNF- $\alpha$. American Journal of Physiology. Endocrinology and Metabolism 2005288 E731-E740.

14 Levi-Montalcini R. The nerve growth factor 35 years later. Science 1987237 1154-1162.
15 Barde YA. The nerve growth factor family. Progress in Growth Factor Research 19902 237-248.

16 Thoenen H, Bandtlow C \& Heumann R. The physiological function of nerve growth factor in the central nervous system: comparison with the periphery. Reviews of Physiology, Biochemistry and Pharmacology 1987109 145-178.

17 Levi-Montalcini R. Effects of mouse tumor transplantation on the nervous system. Annals of the New York Academy of Sciences 1952 55 330-543.

18 Aloe L, Simone MD \& Properzi F. Nerve growth factor: a neurotrophin with activity on cells of the immune system. Microscopic Research and Technique 199945 285-291.

19 Vega JA, Garcia-Suarez O, Hannestad J, Perez-Perez M \& Germana A. Neurotrophins and the immune system. Journal of Anatomy 2003203 1-19.

20 Dicou E, Masson C, Jabbour W \& Nerriere V. Increased frequency of NGF in sera of rheumatoid arthritis and systemic lupus erythematosus patients. Neuroreport 19935 321-324.

21 Bonini SE, Lambiase A, Bonini ST, Angelucci F, Magrini L, Manni L \& Aloe L. Circulating nerve growth factor levels are increased in humans with allergic diseases and asthma. PNAS 199693 10955-10960.

22 Azar ST, Major SC \& Safieh-Garabedian B. Altered plasma levels of nerve growth factor and transforming growth factor$\beta 2$ in type-1 diabetes mellitus. Brain, Behavior, and Immunity 199913 361-366.

23 Gadient RA, Cron KC \& Otten U. Interleukin-1 and tumor necrosis factor synergistically stimulate nerve growth factor (NGF) release from cultured rat astrocytes. Neuroscience Letters $1990117335-340$.

24 Friedman WJ, Larkfors L, Ayer-Lelievre C, Ebendal T, Olson L \& Persson H. Regulation of $\beta$-nerve growth factor expression by inflammatory mediators in hippocampal cultures. Journal of Neuroscience Research 199027 374-382.

25 Bulló M, Peeraully MR \& Trayhurn P. Stimulation of NGF expression and secretion in 3T3-L1 adipocytes by prostaglandins PGD2, PGJ2 and Delta12-PGJ2. American Journal of Physiology. Endocrinology and Metabolism 2005289 E62-E67.

26 Chaldakov GN, Stankulov IS, Fiore M, Ghenev PI \& Aloe L. Nerve growth factor levels and mast cells distribution in human coronary atherosclerosis. Atherosclerosis 2001159 57-66.

27 Chaldakov GN, Fiore M, Stankulov IS, Hristova M, Antonelli A, Manni L, Ghenev PI, Angelucci F \& Aloe L. NGF, BDNF, Leptin, and mast cells in human coronary atherosclerosis and metabolic syndrome. Archives of Physiology and Biochemistry 2001109 357-360.

28 Chaldakov GN, Fiore M, Stankulov IS, Manni L, Hristova MG, Antonelli A, Ghenev PI \& Aloe L. Neurotrophin presence in human coronary atherosclerosis and metabolic syndrome: a role for NGF and BDNF in cardiovascular disease? Progress in Brain Research 2004146 279-289.

29 http://www.eatlas.idf.org/Obesity_and_type_2_diabetes/.

30 National Cholesterol Education Program, Detection, Evaluation and Treatment of High Blood Cholesterol in Adults (Adult Treatment Panel III). NIH Publication, No. 01-3670 May 2001.

31 Segal KR, Van Loan M, Fitzgerald PI, Hodgdon JA \& Van Itallie TB. Lean body mass estimation by bioelectrical impedance analysis: a four-site cross-validation study. American Journal of Clinical Nutrition $1988 \mathbf{4 7} 7-14$.

32 Lang UE, Gallinat J, Danker-Hopfe H, Bajbouj M \& Hellweg R. Nerve growth factor serum concentrations in healthy human voklunteers: physiological variance and stability. Neuroscience Letters $2003 \mathbf{3 4 4} 13-16$.

33 Kerschensteiner M, Gallmeier E, Behrens L, Leal VV, Misgeld T, Klinkert WE, Kolbeck R, Hoppe E, Oropeza-Wekerle RL, Bartke I, Stadelmann C, Lassmann H, Wekerle H \& Hohlfeld R. Activated human $\mathrm{T}$ cells, $\mathrm{B}$ cells, and monocytes produce brain-derived neurotrophic factor in vitro and in inflammatory brain lesions: a neuroprotective role of inflammation? Journal of Experimental Medicine 1999189 865-870. 
34 Moalem G, Gdalyahu A, Shani Y, Otten U, Lazarovici P, Cohen IR \& Schwartz M. Production of neurotrophins by activated T cells: implications for neuroprotective autoimmunity. Journal of Autoimmunity 200015 331-345.

35 Nocker WA \& Renz H. Neurotrophins in clinical diagnostics: pathophysiology and laboratory investigation. Clinica Chimica Acta $200535249-74$.

36 Despres JP, Lemieux I \& Prud'homme D. Treatment of obesity: need to focus on high risk abdominally obese patients. BMJ 2001322 716-720.

37 Hube F, Lietz U, Igel M, Jensen PB, Tornqvist H, Joost HG \& Hauner H. Difference in leptin mRNA levels between omental and subcutaneous abdominal adipose tissue from obese humans. Hormone and Metabolic Research 199628 690-693.

38 Luke AH, Rotimi CN, Cooper RS, Long AE, Forrester TE, Wilks R, Bennett FI, Ogunbiyi O, Compton JA \& Bowsher RR. Leptin and body composition of Nigerians, Jamaicans, and US blacks. American Journal of Clinical Nutrition 199867 391-396.

39 García-Lorda P, Bulló M, Vila R, del Mar Grasa M, Alemany M \& Salas-Salvadó J. Leptin concentrations do not correlate with fat mass nor with metabolic risk factors in morbidly obese females. Diabetes, Nutrition and Metabolism 200114 329-336.

40 Martocchia A, Sigala S, Proietti A, D’Urso R, Spano PF, Missale C \& Falaschi P. Sex-related variations in serum nerve growth factor concentration in humans. Neuropeptides 200236 391-395.

41 Hardardottir I, Grunfeld C \& Feingold KR. Effects of endotoxin and cytokines on lipid metabolism. Current Opinion in Lipidology 1994 $5207-215$.
42 Nonogaki K, Moser AH, Shigenaga J, Feingolf KR \& Grunfeld C. $\beta$-nerve growth factor as a mediator of the acute phase response in vivo. Biochemical and Biophysical Research Communications 1996 219 956-961.

43 Nisoli E, Tonello C, Benarese M, Liberini P \& Carruba MO. Expression of nerve growth factor in brown adipose tissue: implications for thermogenesis and obesity. Endocrinology 1996 137 495-503.

44 Ribases M, Gratacos M, Fernandez-Aranda F, Bellodi L, Boni C, Anderluh M, Cavallini MC, Cellini E, Di Bella D, Erzegovesi S, Foulon C, Gabrovsek M, Gorwood P, Hebebrand J, Hinney A, Holliday J, Hu X, Karwautz A, Kipman A, Komel R, Nacmias B, Remschmidt H, Ricca V, Sorbi S, Wagner G, Treasure J, Collier DA \& Estivill X. Association of BDNF with anorexia, bulimia and age of onset of weight loss in six European populations. Human Molecular Genetics 200413 1205-1212.

$45 \mathrm{Ng}$ TB \& Wong CM. Epidermal and nerve growth factor manifest antilipolytic and lipogenic activities in isolated rat adipocytes. Comparative Biochemistry and Physiology $1985 \mathbf{8 1}$ 687-689.

$46 \mathrm{Bu} \mathrm{G}$, Sun Y, Schwartz AL \& Holtzman DM. Nerve growth factor induces rapid increases in functional cell surface low density lipoprotein receptor-related protein. Journal of Biological Chemistry 1998273 13359-13365.

Received 1 December 2006

Accepted 17 June 2007 